\title{
Induction of Resveratrol Biosynthesis in Grape Skins and Leaves by Ultrasonication Treatment
}

\author{
Md. Mohidul Hasan and Kwang-Hyun Baek ${ }^{*}$ \\ School of Biotechnology, Yeungnam University, Gyeongsan 712-749, Korea
}

\begin{abstract}
Grapes (Vitis vinifera) are one of the most important fruits worldwide and are eaten raw or after conversion to jelly, jam, juice and wine. Grape skins are a major source of resveratrol $\left(3,5,4^{\prime}\right.$-trihydroxystilbene), which has the ability to reduce blood sugar as well as anticancer, anti-inflammatory, and other beneficial cardiovascular effects. In this study, we investigated the increased accumulation of resveratrol in grape skin and leaves following ultrasonication treatment, which has been shown to induce resveratrol accumulation in several plants. Various ultrasonication treatment times and incubation periods were employed to identify the optimum conditions for the maximum accumulation of resveratrol. Treatment and further incubation led to increased resveratrol in both grape skins and leaves, with the highest increases of 7.7-fold and 1.9-fold occurring in response to $5 \mathrm{~min}$ ultrasonication treatment followed by 6 hour incubation and 15 min ultrasonication treatment followed by 3 hour incubation, respectively. The underlying mechanism for the increased amounts of resveratrol were studied by employing a semi-quantitative RT-PCR to monitor the expression levels of the resveratrol synthase $(R S)$ gene in response to ultrasonication treatment. The $R S$ gene increased the expression in response to ultrasonication treatment, suggesting that up-regulation of the $R S$ gene by ultrasonication treatment triggers increased amounts of resveratrol. Taken together, these data indicate that this simple ultrasonication treatment of grapes can be an efficient post-harvest technology for increasing resveratrol in grape skins in addition to cleaning the fruits.
\end{abstract}

Additional key words: elicitation, external stimulus, post-harvest, stilbene

\section{Introduction}

Grapes (Vitis vinifera) are one of the most important fruits cultivated worldwide and can be eaten raw or after being made into jelly, jam, juice and wine. Grape fruits contain a variety of healthy substances including anthocyanin and other phenolic compounds (Cantos et al., 2002). Grape skin is a major source of the potent phytoalexin resveratrol $\left(3,5,4^{\prime}\right.$ -trihydroxystilbene) (Langcake and Pryce, 1976). Grape wine processed from grape skins contains resveratrol, which plays an important role in the French paradox due to the beneficial cardiovascular effects it imparts (Goldberg et al., 1996; Lamuela-Ravento's and Waterhouse, 1993; Pezet and Cuenat, 1996). Resveratrol has been accepted as an important compound for health promotion and prevention of various cancers, cardiovascular disease, and atherosclerosis (Baur and Sinclair, 2006; Delmas et al., 2006; Roupe et al., 2006). Resveratrol also acts as an anti-aging compound by activating the longevity assurance gene, Sirtuin (SIRTI) (Borra et al., 2005). In addition to its availability to markets as an herbal or dietary supplement in tablets, capsules, powders, and extracts from unprocessed botanical supplies (e.g., grape seeds/skins), more up to date applications for resveratrol are starting to include this popular phytochemical infortified foods and beverages.

As one of the most important stilbenephytoalexins, resveratrolis formed or induced in plants as a part of their resistance mechanisms against pathogens, UV irradiation, and many abiotic stresses (Dixon, 2001). Resveratrol is a stilbene compound formed by the Shikimic pathway that has one molecule of $p$-courmaroyl-CoA and three molecules of malonylCoA (Fig. 1). Resveratrol has two isomeric forms, cis- and trans-resveratrol; however, only trans-isomer has been shown to be present in grapes (Jeandet et al., 1991; Langcake and Pryce, 1976; Vrhovsek et al., 1997).

In grape plants, resveratrol is mainly present in grape skins, but also in the seeds (Pezet and Cuenat, 1996), stems (Bavaresco et al., 1997) and leaves (Park et al., 2009). In grape leaves, resveratrol works as a phytoalexin, its formation

\footnotetext{
*Corresponding author: khbaek@ynu.ac.kr

※ Received 19 December 2012; Revised 21 February 2013; Accepted 14 March 2013. This research was supported by a grant (NRF-20110008199) from the Basic Science Research Program through the National Research Foundation (NRF) of Korea.
} 


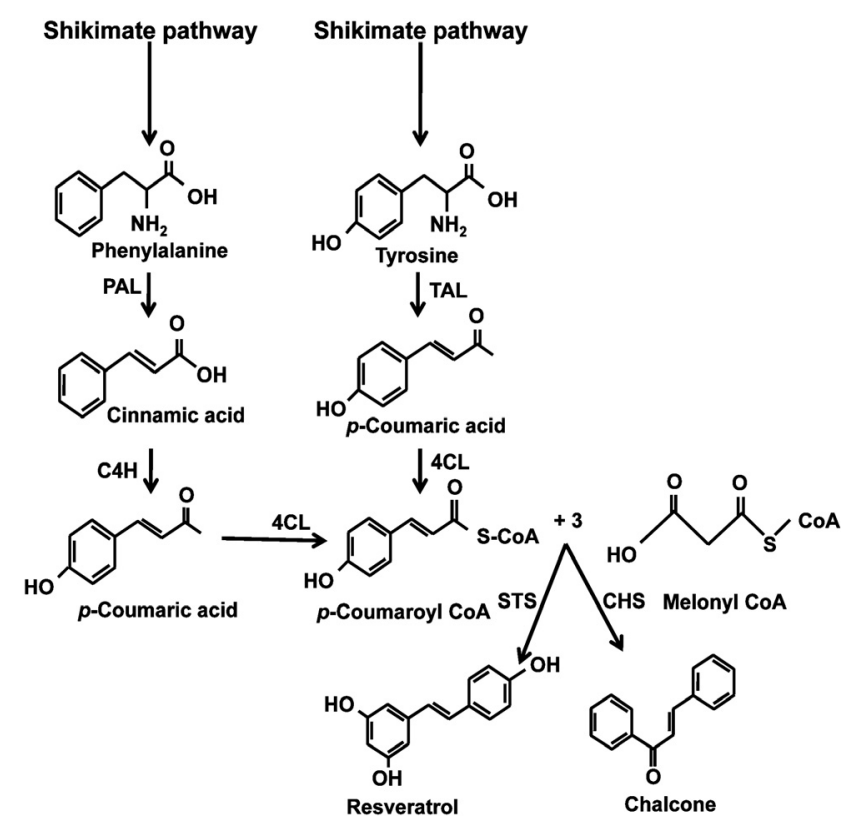

Fig. 1. Biosynthesis pathway of resveratrol. Resveratrol can be synthesized either starting with phenylalanine or from tyrosine. $\mathrm{PAL}$, phenylalanine ammonia lyase; TAL, tyrosine ammonia lyase; $\mathrm{C} 4 \mathrm{H}$, cinnamic acid 4-hydroxylase; 4CL, 4-coumarate: CoA ligase; STS, stilbene synthase/resveratrol synthase; $\mathrm{CHS}$, chalcone synthase.

is correlated with disease resistance and it is actually the most important antifungal compound present (Gerats and Martin, 1992; Jeandet et al., 1991, 1995; Langcake, 1981; Langcake and McCarthy, 1979; Langcake and Pryce, 1976). In grape clusters, stilbenes are located in skin (Jeandet et al., 1991) and are mainly present in the glucosylated form (Creasy et al., 1998; Roggero et al., 1995). The concentration of resveratrol in the skin of ripening grapes and leaves varies considerably depending on the variety and growth stages (Okuda and Yokotosuka, 1996; Park, 2009). In addition, the amount of resveratrol varies widely depending on several factors including the genotype, geographical area of cultivation, climate, growing conditions, and post-harvest operations (Langcake and Pryce, 1976). Many of these factors have been investigated to enable the maximum production of resveratrol (Bavaresco, 2003). Different biotic and abiotic elicitors induce grape resveratrol (Bavaresco et al., 2001), and elicitation of resveratrol-related compounds in grape fruits has been reported in response to fungal infection caused by Botrytis cinerea (Jeandet et al., 1995), Rhizopus stolonifer (Sarig et al., 1997), and Aspergillus spp. (Bavaresco, 2003). Resveratrol can also be elicited by abiotic factors such as UV-C illumination (Cantos et al., 2001, 2002), ozone (GonzalezBarrio et al., 2006), and methyl jasmonate (Vezzulli et al., 2007).

Ultrasonic cleaners are used to clean a wide variety of objects by applying high frequency sound waves to agitate a material suspended in a liquid, and an ultrasonic cleaner has been designed to clean and disinfect all residues of contaminants on fruits and vegetables (Lin and Erel, 1992). This system facilitates removal of all residues, including mud, mold, worms, fungi, bacteria, and agrochemicals. The device uses ultrasound (usually at $20-400 \mathrm{kHz}$ ) and an appropriate cleaning solvent, which is usually tap water. In addition to the cleaning effect, ultrasonication is also used to induce plant secondary metabolites. Indeed, ultrasonicationed to a $60-70 \%$ increase in shikonin yield in Lithospermum erythrorhizon cell culture (Lin and $\mathrm{Wu}, 2001$ ), $75 \%$ increase in ginsenoside saponins in ginseng cells (Lin et al., 2001), and a three-fold increase in taxol in Taxus baccata cell culture (Rezaei et al., 2011). The elicitation of resveratrol by ultrasonication has been extensively studied in whole or sliced peanut kernels, and increases of 8 to 143 times were reported (Potrebko and Resurreccion, 2009; Rudolf and Resurreccion, 2005; Sales and Resurreccion, 2009).

Since grape skin is the major source of resveratrol in wine and juice, induction of high levels of resveratrol in grape skin can have practical applications. Therefore, this study was conducted to determine if ultrasonication of post-harvested grape fruits might have the benefit of increasing resveratrol in the skins in addition to thoroughly cleaning the fruit. To accomplish this, the effects of various ultrasonication treatment times followed by several hours of incubation on the contents of resveratrol in post-harvested grape fruits and leaves were investigated. The results showed that increased amounts of resveratrol in grape fruits and leaves in response to ultrasonication were positively correlated with the up-regulation of $R S$ mRNA, indicating a regulatory mechanism of the gene expression by ultrasonication.

\section{Materials and Methods}

\section{Grape Samples and Chemicals}

The fruits and leaves of the grape Campbell Early cultivar (Viti slabrusca $\times V$. vinifera) were sampled and experiments were conducted two times, in September, 2011 and 2012. Similar-sized grape fruits and leaves were collected from the vineyard of Yeungnam University on a sunny day. Additionally, trans-resveratrol was purchased from SigmaAldrich (St. Louis, MO, U.S.A) and stored in the dark to avoid conversion to $c i s$-resveratrol. All solvents used for extraction and high-performance liquid chromatography (HPLC) analysis were of analytical grade and obtained from SigmaAldrich or Merck (Darmstadt, Germany).

\section{Ultrasonication Treatment}

Grape bunches and leaves harvested from the vineyard 
were treated by ultrasonication in an ultrasonic cleaner with a frequency of $40 \mathrm{kHz}$ frequency (Branson, Model-8510, overall dimensions: $24^{\prime \prime} \times 18^{\prime \prime} \times 14.5^{\prime \prime}$, tank dimensions: $19.5^{\prime \prime} \times 11.5^{\prime \prime} \times 6^{\prime \prime}$, weight: $36 \mathrm{lbs}$, Fredericksburg VA, USA). The bunches and leaves of grapes were placed under sterile $\mathrm{ddH}_{2} \mathrm{O}$ in the ultrasonic tank as described by Lin et al. (2001). The ultrasonication times were adjusted to 5,10 and $15 \mathrm{~min}$ and were followed by incubation for $0,3,6$ and 12 hat $25^{\circ} \mathrm{C}$ in the dark for each ultrasonication time. All samples were analyzed in triplicate. After treatment, as much of the water remaining on the bunches and leaves was removed as much as possible using a paper towel. The control grape bunches and leaves were subjected to the same conditions as the treatment samples, excluding the ultrasonication treatment. Following incubation, the grape skins were peeled removed from the grape fruits, snap-frozen in liquid nitrogen and stored at $-80^{\circ} \mathrm{C}$ and freeze-dried. Ultrasonication application and all analyses were conducted in dim light to prevent trans-resveratrol to cis-resveratrol is omerization as described by Trela et al. (1996).

\section{Resveratrol Analysis}

Extraction of resveratrol from grape skin and leaves was conducted according to the method described by Romero-Perez et al. (2001), with minor modification. Briefly, one gram of freeze-dried grape skins and leaves was ground with a mortar-pestle and then added to $25 \mathrm{~mL}$ of ethanol/water solution $(80: 20 \mathrm{v} / \mathrm{v})$ and vortexed for $30 \mathrm{~s}$. The mixtures were then maintained at $50^{\circ} \mathrm{C}$ for 60 min with gentle stirring. This procedure was repeated two times, with the supernatants being pooled after centrifugation at 3,500 $\mathrm{g}$ for $10 \mathrm{~min}$. The supernatant was subsequently dried using a rotary evaporator, re-dissolved in $5 \mathrm{~mL}$ HPLC grade methanol, and filtered through a $0.45 \mu \mathrm{m}$ syringe filter. Finally, the extracts were injected into a HPLC system.

The reversed-phase HPLC system consisted of a pump equipped with an Eclipse XDB-C18 column (5 $\mu \mathrm{m}, 4.6-250$ $\mathrm{mm}$, Agilent Technologies, USA) and a UV detector (Young-
Lin, YL9100, Seoul, Korea). The isocratic mobile phase contained acetonitrile/water with $0.1 \%$ TFA $(25: 75, \mathrm{v} / \mathrm{v})$ and was applied at a flow rate of $1 \mathrm{~mL} \cdot \mathrm{min}^{-1}$. The wavelength and column temperature were set at $320 \mathrm{~nm}$ and at $40^{\circ} \mathrm{C}$, respectively. The samples were manually injected $(20 \mu \mathrm{L})$ and the peak of resveratrol was identified based on comparison with a standard resveratrol peak. The concentration of resveratrol in the samples was calculated using a regression curve obtained from the peak areas observed during analysis of various concentrations of the standard.

\section{Expression Levels of the Resveratrol Synthase (RS) Gene}

For $R S$ expression level analysis, total RNA was extracted from the grape skins as described by Chang et al. (1993). The expression levels were then measured by semi-quantitative RT-PCR using primers designed based on the published sequences of the grape $R S$ and Actin genes (GenBank accession no. JN858961 and AY680701, respectively). Specifically, primer sets detecting a 160 bp product of $R S$ (forward: GRVF $=5^{\prime}$-GTCCAAGATCACCCATCTTG-3'; reverse: GRVR $=5^{\prime}$ TCCTTAGCAGTTCGAAGGAC-3') and 161 bp product of Actin (forward: GActF = 5'-GATGGTGTGAGTCACACTGT-3'; reverse: GActR $=5^{\prime}$-CGGACAATTTCCCGTTCAGC-3') were used for RT-PCR. Reverse transcription was carried out at $42^{\circ} \mathrm{C}$ for $5 \mathrm{~min}$, followed by PCR amplification of $R S$ for 25 cycles and Actin for 22 cycles of denaturation at $95^{\circ} \mathrm{C}$ for $10 \mathrm{~s}$, annealing at $52^{\circ} \mathrm{C}$ for $40 \mathrm{~s}$, and extension at $72^{\circ} \mathrm{C}$ for $40 \mathrm{~s}$, with a final extension at $72^{\circ} \mathrm{C}$ for $5 \mathrm{~min}$ using Titanium One-Step RT-PCR Kit (Takara Bio Inc., Japan) and an XP Thermal Cycler (BIOER, China). The amplified PCR products were then visualized on $1.2 \%$ agarose gel.

\section{Measurement of Total Soluble Solids in Fruits}

Total soluble solids in both control grapes and those treated by $5 \mathrm{~min}$ sonication followed by $0,3,6$ and 12 $\mathrm{h}$ incubation were measured without peeling off the skins using a refractometer (Atago PAL-1 Pocket Refractometer, Japan) and expressed as Brix ( ${ }^{\circ}$ Brix).
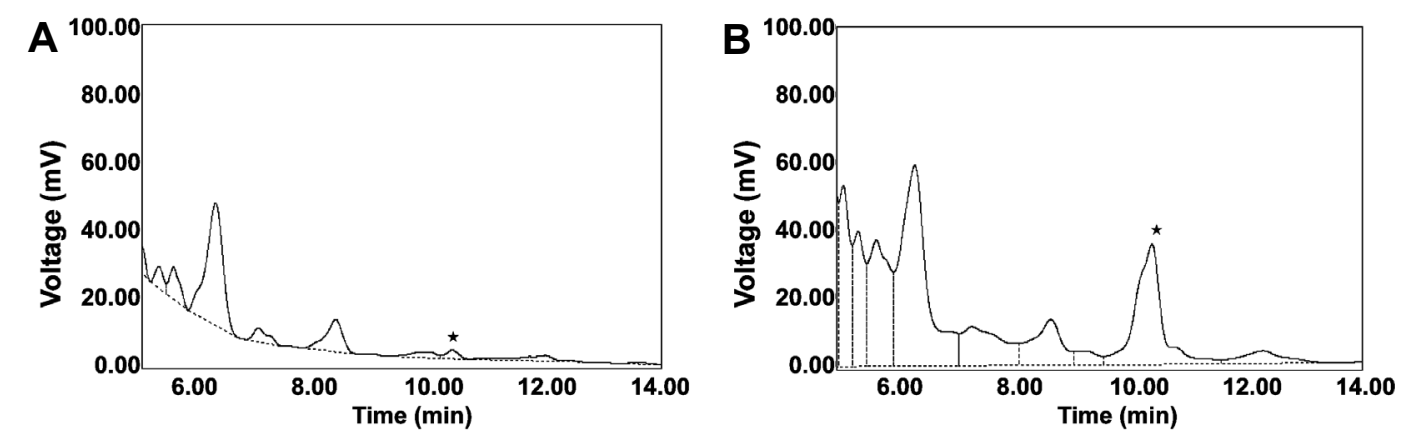

Fig. 2. HPLC chromatogram of the resveratrol in grape skin extracts. Control (A) and ultrasonication for 5 min followed by 6 h incubation (B). 


\section{Statistical Analysis}

Data were analyzed using statistical analysis software (SAS), 2001 version 9.1 (SAS Institute Inc., Cary, NC, USA). Duncan's test was used to determine whether the values were significant $(p<0.01)$.

\section{Results}

\section{Induction of Resveratrol in Grape Skin without Changing} the Sweetness by Ultrasonication Treatment

The resveratrol concentration in grape skins was measured immediately after each ultrasonication treatment (Fig. 3). A significantly higher amount of resveratrol over the control $\left(20.72 \mu \mathrm{g} \cdot \mathrm{g}^{-1}\right)$ was observed following ultrasonication treatment for $5 \min \left(48.20 \mu \mathrm{g} \cdot \mathrm{g}^{-1}\right), 10 \min \left(48.42 \mu \mathrm{g} \cdot \mathrm{g}^{-1}\right)$ and $15 \mathrm{~min}$ $\left(36.92 \mu \mathrm{g} \cdot \mathrm{g}^{-1}\right)$, and ultrasonic treatment for 5 min followed by $6 \mathrm{~h}$ of incubation induced highest levels of resveratrol, with amounts 7.7 -fold higher $\left(148.05 \mu \mathrm{g} \cdot \mathrm{g}^{-1} \mathrm{dw}\right)$ than those in the control $\left(19.18 \mu \mathrm{g} \cdot \mathrm{g}^{-1} \mathrm{dw}\right)$. However, the treatment did not lead to an increase in maintenance of the level of resveratrol. When the amount of reseveratrol was measured

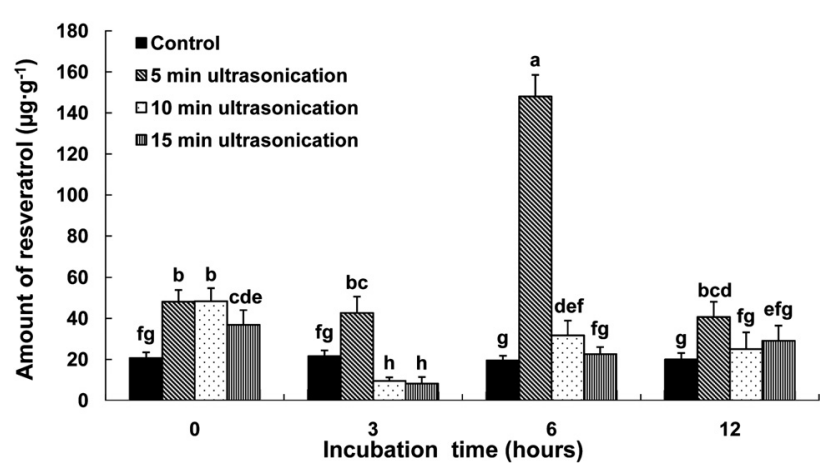

Fig. 3. Amount of resveratrol in grapeskins subjected to different ultrasonication treatments and incubation times. The error bar represents the standard deviation (three replications for each treatment). The same letters indicate no significant difference.

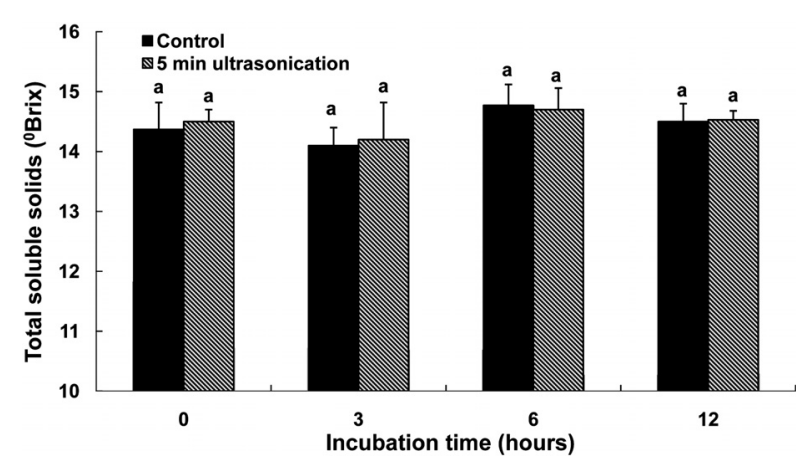

Fig. 4. Amount of total soluble solids in grape fruits treated by 5 min ultrasonication followed by incubation for different times. The error bar represents the standard deviation (three replications for each treatment). The same letters indicate no significant difference. again in fruits incubated for another $6 \mathrm{~h}(5 \mathrm{~min}$ ultrasonication treatment followed by $12 \mathrm{~h}$ ), it had decreased drastically to the level of the samples treated by $5 \mathrm{~min}$ of ultrasonication without any incubation (Fig. 3).

Total soluble solids in the control and the 5 min-ultrasonicated grape fruits were measured to compare the effects of ultrasonication on the sweetness of grape fruits following treatment. The total soluble solids were 14.10 and 14.77 in the control and the treated samples, but there was no significant difference between groups (Fig. 4).

\section{Induction of Resveratrol in Grape Leaves by Ultrasonication Treatment}

Resveratrol accumulation in grape leaves following ultrasonication treatment showed different patterns than those observed in grape fruits. Specifically, ultrasonication for 5 min increased the resveratrol amounts when compared to the control; however, ultrasonication for 10 and $15 \mathrm{~min}$ resulted in reduced amounts of resveratrol when compared to no ultrasonication treatment (Fig. 5). After $3 \mathrm{~h}$ of incubation, leaves subjected to $15 \mathrm{~min}$-ultrasonication accumulated the highest amounts of resveratrol $\left(67.99 \mu \mathrm{g} \cdot \mathrm{g}^{-1} \mathrm{dw}\right)$, as indicated by amounts 1.9 fold higher than those of the control (36.83 $\left.\mu \mathrm{g} \cdot \mathrm{g}^{-1} \mathrm{dw}\right)$. The resveratrol contents in grape leaves subjected to ultrasonication for $5 \mathrm{~min}$ increased at all incubation times (including no incubation), but the levels were not significantly different from those of the controls (Fig. 5).

\section{Up-regulation of Resveratrol Synthase by Ultrasonication Treatment}

Semi-quantitative RT-PCR was conducted to identify the underlying mechanism leading to the increased amounts of resveratrol in ultrasonicated grape fruits and leaves. The total RNA was extracted from the skin of grape fruits that were ultrasonicated for $5 \mathrm{~min}$ following all incubation times, as well as from grape leaves subjected to 15 min ultrasonication

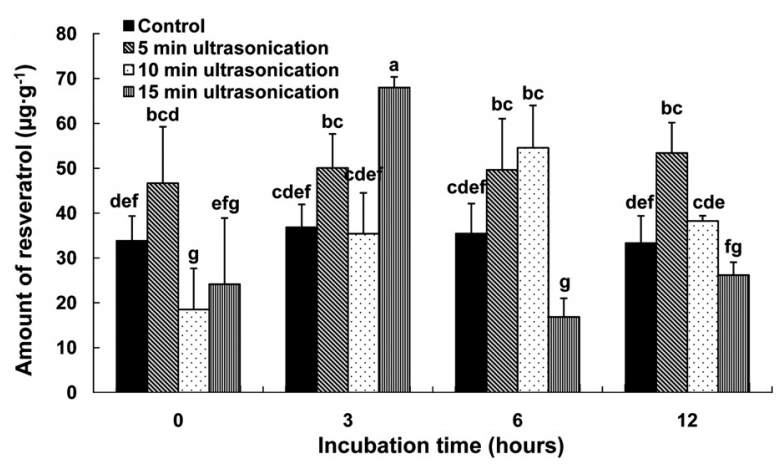

Fig. 5. Amount of resveratrol in grape leaves subjected to different ultrasonication and incubation times. The error bar represents the standard deviation (three replications for each treatment). The same letters indicate no significant difference. 


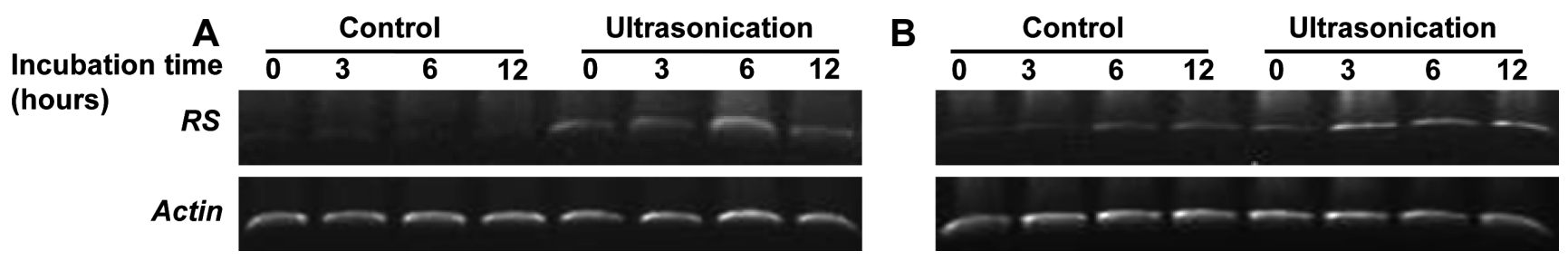

Fig. 6. Semi-quantitative RT-PCR for detection of the relative expression of the resveratrol synthase (RS) gene in ultrasonicated grapeskins (A) and grape leaves (B). Total RNA was extracted from grapeskin and grape leaves subjected to 5 min and 15 min ultrasonication treatment or no ultrasonic treatment, followed by incubation for various lengths of time. The PCR products of the $R S$ and Actin gene were visualized on $1.2 \%$ agarose gels.

and all incubation times. In addition, the Actin gene was amplified to verify equal loading of the total RNA (Fig. 6). In grape skins, the expression levels of $R S$ were low and almost not-detectable; however, they were up-regulated after only $5 \mathrm{~min}$ of ultrasonication treatment and maintained at high values for all incubation times (Fig. 6A). In grape leaves, the $R S$ gene was expressed at very low levels in the leaves. Gene expression in leaves was not changed in response to $15 \mathrm{~min}$ of ultrasonication; however, it was highly up-regulated after $3 \mathrm{~h}$ and further incubation (Fig. 6B).

\section{Discussion}

To the best of our knowledge, increased accumulation of resveratrol in grape skins and leaves by ultrasonication treatment has not been reported to date; although, ultrasonication treatments are usually used to clean grapes. In this study, we applied a one-time ultrasonication treatment to grape fruits and leaves followed by various incubation periods. We observed higher accumulation of resveratrol in grape skins and leaves, though the accumulation patterns and the effective treatments differed among sample types. Specifically, grape skins accumulated very high amounts of resveratrolin response to the treatment, with a 7.7 fold increase being observed in response to $5 \mathrm{~min}$ ultrasonication treatment followed by $6 \mathrm{~h}$ incubation (Fig. 3). In grape leaves, the accumulation was highest after 15 min of ultrasonication treatment followed by $3 \mathrm{~h}$ incubation, with values 1.8 fold higher than those in the control being observed (Fig. 5). There were also increase and decrease in the amounts of resveratrol in response to ultrasonication treatment and incubation time reported in peanut kernels. Specifically, a significant increase in resveratrol concentrations was shown in ultrasonication-treated peanut kernels after $24 \mathrm{~h}$ of incubation in the dark, but a decrease occurred after incubation for 24-36 h (Rudolf and Resurreccion, 2005).

Further analysis of the mechanism of the increase of resveratrolin response to simple ultrasonication treatment was conducted using semi-quantitative RT-PCR to measure expression of the $R S$ gene (Fig. 6). In grape skin, the expression levels of $R S$ increased directly in response to $5 \mathrm{~min}$ of ultrasonication treatment and were then maintained during all observed $12 \mathrm{~h}$ incubation times. Expression of the $R S$ gene in grape leaves did not change in response to $15 \mathrm{~min}$ of ultrasonication, but did increase after $3 \mathrm{~h}$, which was concurrent with the highest resveratrol amounts being observed in leaves after $3 \mathrm{~h}$ incubation following the 15 min-ultrasonication treatment. These data suggest that the accumulation of grape resveratrol in response to ultrasonication occurs in a timedependent manner based on induction of the $R S$ gene. These findings are supported by those of Wang et al. (2010), who found that UV-C treated grapes leaves showed the maximum induction of resveratrol and stilbene synthase after incubation for 16 and $24 \mathrm{~h}$, followed by a decrease after subsequent incubation. The highest expression of $R S$ mRNA was observed in peanut leaves following incubation for 12 to $24 \mathrm{~h}$ after treatment with different biotic and abiotic stresses including yeast extract, paraquat, UV irradiation, ethylene, salicylic acid and methyl jasmonate, which also coincides with our findings (Chung et al., 2003). Although we did not test the enzymatic activity of phenylalanine ammonia-lyase (PAL), the enhanced enzymatic activity of PAL in response to ultrasonication could also be attributed to increased amounts of resveratrol. Ultrasonication treatments of Panax ginseng cells caused a dramatic increase in the enzymatic activity of PAL (Wu and Lin, 2002), which is responsible for deamination of phenylalanine for the biosynthesis of resveratrol precursor coumaryl CoA (Soleas et al., 1997). A coordinated induction of PAL and stilbene synthase activity were observed in response to UV irradiation in leaves of various species of Vitaceae (Fritzemeier and Kindl, 1981) and infungal cell walls in cell suspension cultures of Vitis cv. Optima (Melchior and Kindl, 1991), where the activities of both enzymes were induced in parallel, reaching a maximum after different lengths of time and then decreasing.

Over the past decade, ultrasonication has been effectively employed to induce the production of remarkable secondary metabolites (Cravotto and Cintas, 2006). The biosynthesis 
of ginsenoside in ginseng cells was upregulated by $75 \%$ when compared to a control by simple ultrasonication treatment. This increase might have been due to mechanical stress as well as micro-streaming induced by acoustic cavitations (Lin et al., 2001). A significant enhancement of the trans-esterification of glucose and divinyldicarboxylates by ultrasonication treatment was observed with higher yields than those observed in response to shaking for the same reaction time (Xiao et al., 2005). It is assumed that the effects of ultrasonication occur via increased mass transport owing to the effects of cavitations, which results in faster product dispersion from the enzymatic location (Xiao et al., 2005).

Quantitative measurement of total soluble solids in grapes is an important factor for post-harvest processing and ensuring high quality wine and juice production. Therefore, we also measured the amount of total soluble solids in grape fruits subjected to $5 \mathrm{~min}$ ultrasonication treatment followed by incubation for different times. We found no significant change in response to treatment (Fig. 4), confirming that ultrasonication treatment did not have any negative effects on post-harvest processing of grapes with ultrasonication.

Quality is one of the most important aspects of fresh fruits in the market. Consumers are very concerned with the source of the fruits, existence of hormones, toxic residues, toxins and preservatives used during production and post-harvest operations. The preservation and quality of grapes are directly related to the cultivars, crop conditions, cultivation system and post-harvest operations. Post-harvest cleaning of grape fruits by ultrasonication treatment maybe the best option for eliminating all debris from the grapes. Another advantage of this technique is the improved nutrients, especially the phytochemical resveratrol. This technique also did not show any negative effects on the amount of total soluble solids in grapes; therefore, it has practical importance in enology and in the food industry because the high levels of resveratrol in grape skin can be applied for production of resveratrol-rich wine, juice and jams. Further investigation is required to determine the proper ultrasonication treatment strength and incubation time for maximization of resveratrol production in grape skins.

\section{Literature Cited}

Baur, J.A. and D.A. Sinclair. 2006. Therapeutic potential of resveratrol: the in vivo evidence. Nat. Rev. Drug Disc. 5: 493-506.

Bavaresco, L. 2003. Role of viticultural factors on stilbene concentrations of grapes and wine. Drugs Experimental Clinical Res. 29:181-187.

Bavaresco, L., D. Petegolli, E. Cantú, M. Fregoni, G. Chiusa, and M. Trevisan. 1997. Elicitation and accumulation of stilbene phytoalexins in grape-vine berries infected by Botrytis cinerea. Vitis 36:77-83.

Bavaresco, L., S. Pezzutto, A. Ragga, F. Ferrari, and M. Trevisan. 2001. Effect of nitrogen supply on trans-resveratrol concentration in berries of Vitis vinifera L. cv. Cabernet Sauvignon. Vitis 40:229-230.

Borra, M.T., C. Smith, and J.M. Denu. 2005. Mechanism of human SIRT1 activation by resveratrol. J. Biol. Chem. 280:17187-17195.

Cantos, E., J.C. Espín, and F.A. Tomás-Barberán. 2001. Postharvest induction modeling method using UV irradiation pulses for obtaining resveratrol-enriched table grapes: A new "functional" fruit? J. Agric. Food Chem. 49:5052-5058.

Cantos, E., J.C. Espín, and F.A. Tomás-Barberán. 2002. Postharvest stilbene-enrichment of red and white table grape varieties using UV-C irradiation pulses. J. Agric. Food Chem. 50:6322-6329.

Chang, S., J. Puryear, and J. Cairney. 1993. A simple and efficient method for isolating RNA from pine trees. Plant Mol. Biol. Rep. 11:113-116.

Chung, I.-M., M.R. Park, J.C. Chun, and S.J. Yun. 2003. Resveratrol accumulation and resveratrol synthase gene expression in response to abiotic stresses and hormones in peanut plants. Plant Sci. 164:103-109.

Cravotto, G. and P. Cintas. 2006. Power ultrasound in organic synthesis: moving cavitational chemistry from academia to innovative and large-scale applications. Chem. Soc. Rev. 35:180-196.

Creasy, L.L. and M. Coffee. 1998. Phytoalexin production potential of grape berries. J. Amer. Soc. Hort. Sci. 113:230-234.

Delmas, D., A. Lancon, D. Colin, B. Jannin, and N. Latruffe. 2006. Resveratrol as a chemo preventive agent: A promising molecule for fighting cancer. Curr. Drug Targets. 7:423-442.

Dixon, R.A. 2001. Natural products and plant disease resistance. Nature 411:843-847.

Fritzemeier, K.-H. and H. Kindl. 1981. Coordinate induction by UV light of stilbene synthase, phenylalanine ammonia-lyase and cinnamate 4-hydroxylase in leaves of vitaceae. Planta 151:48-52.

Gerats, A.G.M. and C. Martin. 1992. Flavonoid synthesis in Petunia hybrida: Genetic and molecular biology of flower colour, p. 165-199. In: R.K. Ibrahim and H.A. Stafford (eds.). Recent advances in phytochemistry, Vol. 26. Phenolic metabolism in plants. Kluwer Academic/Plenum Publishers, New York.

Goldberg, D.M., E. Tsang, A. Karumanchiri, E.P. Diamandis, G. Soleas, and E. Ng. 1996. Method to assay the concentrations of phenolic constituents of biological interest in wines. Anal. Chem. 68:1688-1694.

Gonzalez-Barrio, R., D. Beltran, E. Cantos, M.I. Gil, J.C. Espin, and F.A. Tomas-Barberan. 2006. Comparison of ozone and UV-C treatments on the postharvest stilbenoid monomer, dimer, and trimer induction in var. 'Superior' white table grapes. J. Agric. Food Chem. 54:4222-4228.

Jeandet, P., R. Bessis, and B. Gautheron. 1991. The production of resveratrol $\left(3,5,4^{\prime}\right.$-trihydroxystilbene) by grape berries in different developmental stages. Am. J. Enol. Vitc. 42:41-46.

Jeandet, P., R. Bessis, M. Sbaghi, P. Meunier, and P. Trollat. 1995. Resveratrol content of wines of different ages: relationship with fungal disease pressure in the vineyard. Am. J. Enol. 
Vitic. 46:1-4.

Lamuela-Ravento's, R.M. and A.L. Waterhouse. 1993. Occurrence of resveratrol in selected California wines by a new HPLC method. J. Agric. Food Chem. 41:521-523.

Langcake, P. 1981. Disease resistance of Vitis spp. and the production of the stress metabolites resveratrol, $\varepsilon$-viniferin, $\alpha$-viniferin and pterostilbene. Physiol. Plant Pathol. 18:213-226.

Langcake, P. and R.J. Pryce. 1976. The production of resveratrol by Vitis vinifera and other members of the vitaceae as a response to infection or injury. Physiol. Plant Pathol. 9:77-86.

Langcake, P. and W.V. McCarthy. 1979. The relationship of resveratrol production to infection of grapevine by Botrytis cinerea. Vitis 18:244-253.

Lin, I. and D. Erel. 1992. Dynamic ultrasonic cleaning and disinfecting device and method. U.S. Patent 5,113,881, filled Jun, 22, 1990, and issued May 19, 1992.

Lin, L. and J. Wu. 2001. Enhancement of shikon in production in single- and two-phase suspension cultures of Lithospermum erythrorhizon cells using low-energy ultrasound. Biotechnol. Bioeng. 78:81-88.

Lin, L., J. Wu, K.-P. Ho, and S. Qi. 2001. Ultrasound-induced physiological effects and secondary metabolite (saponin) production in Panax ginseng cell cultures. Ultrasound Med. Biol. 27:1147-1152.

Melchior, F. and H. Kindl. 1991. Coordinate and elicitor-dependent expression of stilbene synthase and phenylalanine ammonia-lyase genes in Vitis cv. Optima. Arch. Biochem. Biophys. 288:552-557.

Okuda, T. and K. Yokotosuka. 1996. Trans-resveratrol concentrations in berry skins and wines from grapes grown in Japan. Am. J. Enol. Vitic. 47:93-99.

Park, H.J., J.-M. Ko, N.-R. An, Y.S. Kim, and H.-C. Cha. 2009. Contents of Trans-resveratrol in Korean grape cultivars, including "Kyoho". J. Plant Biol. 52:319-324.

Pezet, R. and P. Cuenat. 1996. Resveratrol in wine: Extraction from skin during fermentation and post-fermentation standing of must from Gamay grapes. Am. J. Enol. Vitic. 47:287-290.

Potrebko, I. and A.V.A. Resurreccion. 2009. Effect of ultraviolet doses in combined ultraviolet-ultrasound treatments on transresveratrol and trans-piceid contents in sliced peanut kernels. J. Agric. Food Chem. 57:7750-7756.

Rezaei, A., F. Ghanati, and M.A. Dehaghi. 2011. Stimulation of taxol production by combined salicylic acid elicitation and sonication in Taxus baccata cell culture. Int. Conf. Life Sci. Technol. IPCBEE Vol. 3. IACSIT press, Singapore. p. 193-197.
Roggero, J.-P. and C. Garcia-Parrilla. 1995. Effects of ultraviolet irradiation on resveratrol and changes in resveratrol and various of its derivatives in the skins of ripening grapes. Sci. Aliments 15:411-422.

Romero-Pérez, A.I., R.M. Lamuela-Raventós, C. Andrés-Lacueva, and M.C. de La Torre-Boronat. 2001. Method for the quantitative extraction of resveratrol and piceid isomers in grape berry skin. Effect of powdery mildew on the stilbene content. J. Agric. Food Chem. 49:210-215.

Roupe, K.A., C.M. Remsberg, J.A. Yanez, and N.M. Davies. 2006. Pharmacometrics of stilbenes: Seguing towards the clinic. Curr. Clin. Pharm. 1:81-101.

Rudolf, J.R. and A.V.A. Resurreccion. 2005. Elicitation of resveratrol in peanut kernels by application of abiotic stresses. J. Agric Food Chem. 53:10186-10192.

Sales, J.M. and A.V.A. Resurreccion. 2009. Maximising resveratrol and piceid contents in UV and ultrasound treated peanuts. Food Chem. 11:7674-7680.

Sarig, P., Y. Zutkhi, A. Monjauze, N. Lisker, and R. Ben-Arie. 1997. Phytoalexin elicitation in grape berries and their susceptibility to Rhizopusstolonifer. Physiol. Mol. Plant Pathol. 50:337-347.

Soleas, G.J., E.P. Diamandis, and D.M. Goldberg. 1997. Resveratrol: A molecule whose time has come? and gone? Clinical Biochem. 30:91-113.

Trela, B.C. and A.L. Waterhouse. 1996. Resveratrol: Isomeric molar absorptivities and stability. J. Agric. Food Chem. 44:1253-1257.

Vezzulli, S., S. Civardi, F. Ferrari, and L. Bavaresco. 2007. Methyl jasmonate treatment as a trigger of resveratrol synthesis in cultivated grapevine. Am. J. Enol. Vitic. 58:530-533.

Vrhovsek, U., S. Wendelin, and R. Eder. 1997. Effects of various vinification techniques on the concentration of cis- and transresveratrol and resveratrol glucoside isomers in wine. Am. J. Enol. Vitic. 48:214-219.

Wang, W., K. Tang, H.R. Yang, P.F. Wen, P. Zhang, H.L. Wang, and W.D. Huang. 2010. Distribution of resveratrol and stilbene synthase in young grape plants (Vitis vinifera L. cv. Cabernet Sauvignon) and the effect of UV-C on its accumulation. Plant Physiol. Biochem. 48:142-152.

$\mathrm{Wu}$, J. and L. Lin. 2002. Ultrasound-induced stress responses of Panax ginsengcells: Enzymatic browning and phenolics production. Biotechnol. Prog. 18:862-866.

Xiao, Y.M., Q. Wu, Y. Cai, and X.-F. Lin. 2005. Ultrasoundaccelerated enzymatic synthesis of sugar esters in nonaqueous solvents. Carbohydr. Res. 340:2097-2103. 